\title{
Comparison of Fog Computing \& Cloud Computing
}

\author{
Vishal Kumar $^{\text {a }}$, Asif Ali Laghari ${ }^{\text {b,*, Shahid Karim }}{ }^{\text {c }}$, Muhammad Shakir ${ }^{\mathrm{d}}$, \\ Ali Anwar Brohi ${ }^{\mathrm{e}}$ \\ ${ }^{a}$ School of Control Engineering, Harbin Institute of Technology Harbin, 150001, China \\ ${ }^{b}$ School of Computer Science \& Technology, Harbin Institute of Technology Harbin, 150001, China \\ ${ }^{c}$ School of Information \& Engineering, Harbin Institute of Technology Harbin, 150001, China \\ ${ }^{d}$ School of Control Engineering, Harbin Institute of Technology Harbin, 150001, China \\ ${ }^{e}$ School of Power Engineering, Harbin Institute of Technology Harbin, 150001, China
}

Received: 21 August 2018; Accepted: 05 November 2018; Published: 08 January 2019

\begin{abstract}
Fog computing is extending cloud computing by transferring computation on the edge of networks such as mobile collaborative devices or fixed nodes with built-in data storage, computing, and communication devices. Fog gives focal points of enhanced proficiency, better security, organize data transfer capacity sparing and versatility. With a specific end goal to give imperative subtle elements of Fog registering, we propose attributes of this region and separate from cloud computing research. Cloud computing is developing innovation which gives figuring assets to a specific assignment on pay per utilize. Cloud computing gives benefit three unique models and the cloud gives shoddy; midway oversaw assets for dependable registering for performing required errands. This paper gives correlation and attributes both Fog and cloud computing differs by outline, arrangement, administrations and devices for associations and clients. This comparison shows that Fog provides more flexible infrastructure and better service of data processing by consuming low network bandwidth instead of shifting whole data to the cloud.
\end{abstract}

Index Terms: Cloud computing, Fog computing, Comparison.

(C) 2019 Published by MECS Publisher. Selection and/or peer review under responsibility of the Research Association of Modern Education and Computer Science

\section{Introduction}

High-performance computing trends changed according to technological innovation and user demands [1]. Previously grid computing was a consideration in the service providers and organization for use of free

${ }^{*}$ Corresponding author. Tel.: +86-15636147179;

E-mail address: asiflaghari@hit.edu.cn 
resources for high-performance computing [2]. Currently, the extended version of grid computing is used, named as cloud computing and technology vendors like IBM, Google and Microsoft extending cloud computing with Fog computing. Fog Computing is a progressed or expanded variant of cloud computing where computing happens at the edge of the system. There are numerous organizations as of now spending a considerable measure of research on this point like Cisco and so on. Fog computing defined as in [3], a situation where a support of all of heterogeneous (remote and some of the time self-ruling), universal and decentralized devices convey and conceivably participate between them and with the network to perform capacity and preparing undertakings without the intercession of outsiders. These undertakings can be for supporting essential network capacities or new administrations and applications that keep running in a sandboxed situation. Clients renting some portion of their devices to have these administrations get motivators for doing.

It was assumed by Marketsandmarkets that in the year 2015 cloud or high-performance computing (HPC) produce up to $\$ 4.37$ billion and it will increase up to $\$ 10.83$ billion in 2020 [4]. The future investment was forecasted up to $\$ 6$ trillion for the next five years on IOT solutions and which will also consume on Fog computing the hot topic among the businesses and investors. Technology thinker believes that when Fog computing into the market then data load will not essential for modern cloud computing to carry because IOT with $4 \mathrm{G}$ technology endorsed by the organization to enlarge use for solutions that will make more profit and trustworthy.

Our paper is organized into 5 sections, in section 2 we describe the Fog computing and section 3 is based on cloud computing. Section 4 provides a comparison of Fog and cloud computing; finally, in section 5 we conclude our work.

\section{Fog Computing}

Fog computing is more advanced and its performance better than cloud computing for handling user requests and emerging standards [5]. Cloud computing is infrastructure based and required hardware and software to manage task and processing where Fog utilizes resources of devices on edge but it will not replace cloud computing at present time, which is top of all business and provide employment to the world. It was concluded that cloud computing and Fog computing have own characteristics to process data burdens and preferences but are the supplement to each other [6]. Edge registering assumes a pivotal part of the Internet of Things (IoT) [7]. Security and privacy concerns are also found in the framework of Fog computing which still big issue business point of view as compared to cloud computing. With the help of Fog computing, the ideal model of business will develop which reduce the delay, jitter that will beneficial for fast computing and force to businesses of cloud computing to reduce the cost of processing utilities [8].

Fog computing defined as a progressed or expanded variant of cloud computing where the computing happens at the edge of the system. There are numerous organizations as of now spending a considerable measure of research on this point like Cisco and so on $[9,10]$.

- It is like cloud computing, however, is far denser in land dissemination what's more, area and its nearness to end clients is more, which implies they give a quicker end-client encounter than distributed computing and have better execution.

- The inquiries concerning its unpredictability, pragmatic attainability, cost and execution are emerging in various research groups. There is likewise a noteworthy concern about its dependability or sturdiness in playing out a wide range of operations performed by distributed computing frameworks.

\subsection{Characteristics of Fog Computing}

- Low inertness, edge area and area mindfulness: Fog processing arrangement better administrations to 
end clients at the edge of the network.

- Geographical Distribution: Fog computing application, objective and services are widely distributed.

- Support for Mobility: Fog figuring give versatility systems like decouple have the personality to area character by utilizing LISP protocol.

- Real time interactions: speedy services need real time interaction in Fog computing.

- Heterogeneity: Fog computing supports heterogeneous devices and support nodes in a wide variety of environments.

- Interoperability: Fog gives a wide range of services so for that purpose Fog devices incorporate for streaming of services.

Fog computing is also known as fogging [11]. Fog computing foundation is distributed, few processes are executed at the edge of the network by intelligent devices and remaining part will shift to cloud for processing. Fog is techniques is utilized devices for data processing, storage from client to cloud and provide the fast network with security to transfer low volume of data for processing to cloud and most of the processing accomplish on the edge devices [12].

Smart lighting system working on the set of instructions is an illustration of the Internet of Things [13]. Data processing may interrupt the results that lights are turned on or turned off when there is no movement for time span in IoT. This data and outcomes are best refined at the outskirt.

The organization executing the Smart lighting framework could likewise wish to venture up towards vitality effectiveness and for perceiving about the ideal opportunity for which the lights were turned on? The information which supports this "whole point of view" of how the keen lighting is being actualized would require information to be requested and prepared by an announcing framework keep running in the cloud [14].

\section{Cloud Computing}

Cloud computing is popular as provide services and computing resources on pay per use to users on their demands [15]. Cloud computing is an extension of cluster and grid computing which was used to collect resources at one central place and utilize them to high-performance computing. Cloud computing architecture provides three types of services such as software as a service (SaaS), Platform as a service (PaaS) and Infrastructure as a Service (SaaS) [16, 54]. NIST gives the definition of cloud that design of computing architecture, which provides high powerful resources for computing, storage, the environment of application development with multiple platforms with ease of management and coordination of all devices of resources at one place [17, 18]. Cloud computing also provide mobility features know as mobile cloud computing. Mobile cloud computing is defined as a "new worldview for portable applications, where the information handling and storage are relocated from the nearby clients to intense and centralized computing platforms situated in the clouds" [19]. Cloud computing models are different according to the type of services they provide such as SaaS deals customer services like accounting, database application software and emails but the user did not deal with technical infrastructure of cloud [20, 21]. PaaS provides a platform for application developer like programming environment, by using those tools develop applications and have rights configure and technically manage the cloud [22, 23]. IaaS provides services of the infrastructure of clouds such as manage servers, storage and network devices $[24,25]$. IaaS provides the right to manage, change or configure cloud infrastructure according to their needs. Cloud computing models are given in figure 1.

\subsection{Cloud Deployment Models}

Cloud computing deployed according to four different ways such as public, private, community and hybrid $[26,27]$. 


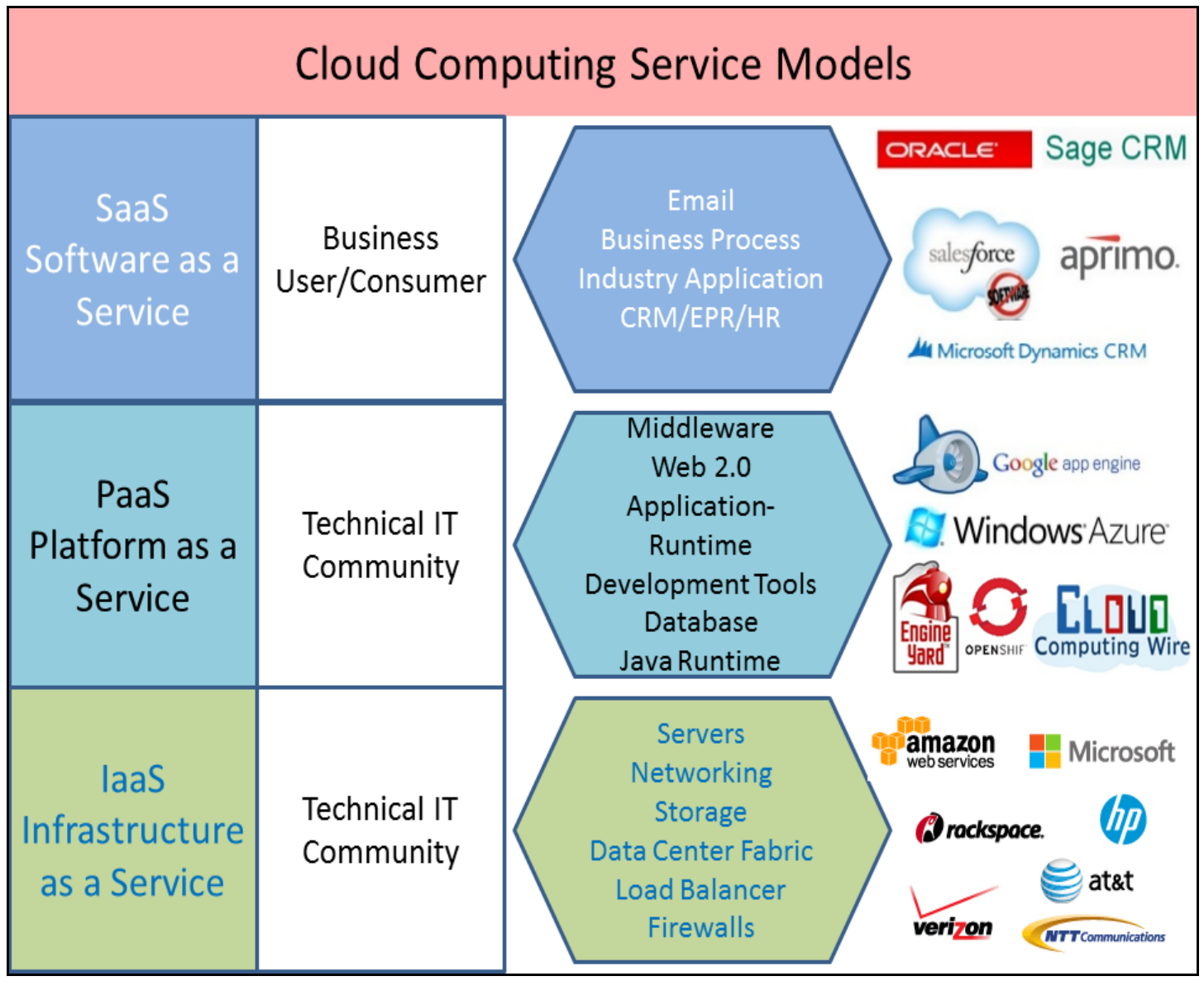

Fig.1. Cloud Computing Service Model [15]

- Public cloud: This design of cloud is open for general public use and is designed and managed by government organizations, educational sector or business organizations or combination of some of them, its depend on the service providers [28].

- Private cloud: Public cloud is designed and developed for the private use of organizations such as education, business and security agencies or managed by multiple organizations for consumers [29].

- Community cloud: the cloud designed and managed by a specific community to use it for security or business purpose. It managed by one or two organizations based on the community [30].

- Hybrid cloud: This foundation depends on the arrangement of multiple cloud designs such as public, community, or private which composed to together for empowers computing resources information and application transportability (e.g., cloud blasting for stack adjusting between clouds) [31, 32].

\section{Comparison}

In this section Fog and cloud computing comparison is given in table 1. 
Table 1. Fog vs Cloud Computing

\begin{tabular}{|c|c|c|}
\hline Parameters & Fog Computing & Cloud Computing \\
\hline Goal & $\begin{array}{l}\text { Enhance proficiency and execution of process that } \\
\text { should be transported to the cloud for handling, } \\
\text { investigation and storage }\end{array}$ & $\begin{array}{l}\text { Give a request of greatness change in } \\
\text { the practical, powerful provisioning of } \\
\text { IT administrations }\end{array}$ \\
\hline Computational focuses & Fog operates on network edge & $\begin{array}{l}\text { Data and applications are processed in } \\
\text { the Cloud }\end{array}$ \\
\hline Abstraction Level & High & High \\
\hline scalability Degree & High & High \\
\hline Support of Multitask & Yes & Yes \\
\hline Level Transparency & High & High \\
\hline Run time & Real time services & Real time services \\
\hline Type of Requests & Lots of High allocation & Lots of small allocation \\
\hline Allocation unit & All shapes and sizes (wide \&narrow) & All shapes and sizes (wide \&narrow) \\
\hline Level of Virtualization & Vital & Vital \\
\hline Accessible type & IP & IP \\
\hline Transmission & Device to device & Device to Cloud \\
\hline Security & Possible, determined & Undefined \\
\hline Infrastructure & Flexible & 3 models (PaaS, IaaS, SaaS) \\
\hline Support of Operating System & hypervisor virtualization & $\begin{array}{l}\text { A hypervisor (VM) on which } \\
\text { multiple OSs run }\end{array}$ \\
\hline Ownership & Multiple & Single \\
\hline Service negotiation & SLA based & SLA based \\
\hline Support of User management & Centralized & $\begin{array}{l}\text { Centralized or can be delegated to third } \\
\text { party }\end{array}$ \\
\hline Resource management & Centralized & Centralized/Distributed \\
\hline Allocation/Scheduling & Centralized & decentralized/centralized \\
\hline Interoperability & Interoperability between heterogeneous resources. & Web Services (SOAP and REST) \\
\hline Failure management & rescheduling of failed tasks & $\begin{array}{l}\text { Strong (VMs can be easily migrated } \\
\text { from one node to other) }\end{array}$ \\
\hline Service price & Utility pricing and Pay per use as go & $\begin{array}{l}\text { Utility pricing, discounted for larger } \\
\text { customers }\end{array}$ \\
\hline Type of service & CPU, network, memory, bandwidth, device, storage & $\begin{array}{l}\text { IaaS, PaaS, SaaS, Everything as a } \\
\text { service }\end{array}$ \\
\hline Example of real world & $\begin{array}{l}\text { Significant Fog applications involve real-time } \\
\text { interactions rather than } 228 \text { batch processing. }\end{array}$ & $\begin{array}{l}\text { Amazon Web Service (AWS), Google } \\
\text { apps }\end{array}$ \\
\hline Response Time & Low & High \\
\hline Critical object & Service & Service \\
\hline Number of users & Unlimited & Unlimited \\
\hline Resource & Unlimited & Unlimited \\
\hline Future & Next Generation of Internet and computing & Fog Computing \\
\hline
\end{tabular}

Comparison table shows that few parameters are same for both Fog and cloud such as portable access, virtualization, multitask, transparency, service negotiation, critical object, number of users support and resources provided by $[33,34,55]$. Rest of other parameters Fog computing provide more advance benefits compare to cloud computing such as Fog computing provide a response in a short time but cloud takes high. Other parameters like request type, transmission, security, user management and resource management, scheduling, interoperability, failure management, pricing of services and type of services of Fog computing are better than cloud computing [35, 36, 37, 38]. Table 2 provide details of Fog and cloud applications details where Table 3 about the tools. 


\section{Open Research Issues}

From one viewpoint, the improvement of Fog and Edge mists incorporates devoted offices, working framework, organize and middleware strategies to manufacture and work such miniaturized scale server farms that host virtualized registering assets [56]. Then again, the utilization of Fog expects the expansion to current programming models and proposes new deliberations that will enable engineers to outline new applications that take advantage from such enormously conveyed frameworks. The utilization of this approach additionally opens up different difficulties in: security and protection (as a client currently needs to "trust" each smaller scale server farm they interface with), bolster for asset administration for versatile clients who exchange session starting with one miniaturized scale server farm then onto the next, and bolster for "installing" such smaller-scale server farms into gadgets (e.g. autos, structures, and so forth.) [57]. By adding quality of experience (QoE) domain in Fog computing, service will be improved for video streaming and game services [58]. The technical parameters are different in every field such as multimedia services contains bitrate of video, frame rate, video codecs etc. and game required high processing power to adding QoE will provide user stratification about the services [59].

To fulfill the consistently expanding interest for Cloud Computing assets from rising applications, for example, Internet-of-Things (IoT), scholastics and industry specialists are currently pushing for going from substantial unified Cloud Computing foundations to smaller scale server farms situated at the edge of the system. These smaller scale server farms are frequently more like a client (geologically and in getting to dormancy) contrasted with the incorporated cloud server center [60, 61]. The point of using such edge assets is to offload calculation that would have "customarily" been completed at the cloud server center to an asset that is more like a client or edge devices. This vision additionally recognizes the variety in arranging dormancy from an end client to cloud server center. Though the system around a server center is frequently high limit and speed that close to the client devices may have fluid properties (Regarding flexibility, transmission capacity, idleness, and so forth). Cloud and network management are important for service providers to provide QoS to end users, so they can evaluate their services via subjective feedback of users, sometimes which contains inaccurate and negative responses [62,63]. Therefore, for proper management accurate and positive QoE is required, leading to further work in QoE monitoring and management.

Table 2. Fog and Cloud Computing Applications

\begin{tabular}{|c|c|c|}
\hline Technology & Applications & Comments \\
\hline \multirow{3}{*}{ Fog } & $\begin{array}{l}\text { Video streaming, gaming } \\
\text { (Rendering and decoding) }\end{array}$ & $\begin{array}{l}\text { It can give huge administrations to video and gaming applications and } \\
\text { empower new stream of intelligent multimedia era. It also provides } \\
\text { rendering and decoding of multimedia contents }[39,40] \text {. }\end{array}$ \\
\hline & $\begin{array}{l}\text { Small cities, environmental } \\
\text { monitoring (Fusion and } \\
\text { Aggregation) }\end{array}$ & $\begin{array}{l}\text { Cisco gives an application store which enables clients to download } \\
\text { applications to the IOx devices and an application administration comfort } \\
\text { which is intended for controlling and observing the execution of an } \\
\text { application. It also provides Geo-Distribution and security [41]. }\end{array}$ \\
\hline & $\begin{array}{l}\text { SCV, smart Grid, Int. } \\
\text { Transportation } \\
\text { (Partitioning/quasi- } \\
\text { autonomy, security) }\end{array}$ & This provides services to federal/state agencies, car manufacturing. \\
\hline \multirow{3}{*}{ Cloud } & $\begin{array}{l}\text { Deep data Mining, Search, } \\
\text { financial algorithm } \\
\text { (MR/Hadoop) }\end{array}$ & $\begin{array}{l}\text { It is used for large batch jobs of data search and analysis and this can be } \\
\text { used by individuals or enterprises }[42,43] \text {. }\end{array}$ \\
\hline & $\begin{array}{l}\text { e-commerce (Web } \\
\text { applications) }\end{array}$ & $\begin{array}{l}\text { This provides services of shopping, transactions of money for enterprises } \\
\text { and also for individuals }[44,45] \text {. }\end{array}$ \\
\hline & Cloudo & $\begin{array}{l}\text { A idle computer System that online on the Internet via web Browser } \\
\text { [46]. }\end{array}$ \\
\hline
\end{tabular}


Table 3. Fog and Cloud Tools

\begin{tabular}{|l|l|l|}
\hline Technology & Tools & Comments \\
\hline \multirow{3}{*}{ Fog } & IFOGSIM & $\begin{array}{l}\text { iFogSim empowers the reproduction of asset administration and application } \\
\text { planning arrangements crosswise over edge and cloud assets under various } \\
\text { situations and conditions [47, 48]. }\end{array}$ \\
\cline { 2 - 3 } & Fog Project & $\begin{array}{l}\text { A free Open-source network computing cloning and management solution } \\
\text { [49]. }\end{array}$ \\
\hline \multirow{3}{*}{ Cloud } & CloudSim & $\begin{array}{l}\text { CloudSim is used by developers for evaluation of the requirements of larg- } \\
\text { scale cloud applications [50]. }\end{array}$ \\
\cline { 2 - 3 } & Zenoss & $\begin{array}{l}\text { A solitary, coordinated item that screens the whole IT framework, wherever } \\
\text { it is conveyed (physical, virtual, or in cloud) [51, 52]. }\end{array}$ \\
\cline { 2 - 3 } & Cloudera & $\begin{array}{l}\text { An open-source Hadoop programming structure is progressively utilized as a } \\
\text { part of cloud computing organizations because of its adaptability with } \\
\text { cluster based, information concentrated questions and different tasks [53]. }\end{array}$ \\
\hline
\end{tabular}

\section{Conclusion}

In this paper, we presented a comparison of Fog and cloud computing this will help to understand the differences between both the researchers. Cloud computing technology now mature and many development tools are available for design and implement cloud infrastructure. Fog computing is an early stage of research and still, prototype models and development tools are under research phase but we believe that Fog computing is future of modern computing technology and evolve very fast and utilized edge of devices for computational resources. The Tables given in paper provides details of advantages which provide of Fog computing over cloud computing. Fog will promising business model for service providers in the future.

\section{References}

[1] Pijanowski, Bryan C., Amin Tayyebi, Jarrod Doucette, Burak K. Pekin, David Braun, and James Plourde. "A big data urban growth simulation at a national scale: configuring the GIS and neural network based land transformation model to run in a high performance computing (HPC) environment." Environmental Modelling \& Software 51 (2014): 250-268.

[2] Bera, Samaresh, Sudip Misra, and Joel JPC Rodrigues. "Cloud computing applications for smart grid: A survey." IEEE Transactions on Parallel and Distributed Systems 26, no. 5 (2015): 1477-1494.

[3] Vaquero, Luis M., and Luis Rodero-Merino. "Finding your way in the fog: Towards a comprehensive definition of fog computing." ACM SIGCOMM Computer Communication Review 44, no. 5 (2014): 2732.

[4] https://www.marketsandmarkets.com/PressReleases/cloud-high-performance-computing.asp

[5] Zhang, Huaqing, Yanru Zhang, Yunan Gu, Dusit Niyato, and Zhu Han. "A Hierarchical Game Framework for Resource Management in Fog Computing." IEEE Communications Magazine 55, no. 8 (2017): 52-57.

[6] Stojmenovic, Ivan. "Fog computing: A cloud to the ground support for smart things and machine-tomachine networks." In Telecommunication Networks and Applications Conference (ATNAC), 2014 Australasian, pp. 117-122. IEEE, 2014.

[7] Rahmani, Amir M., Tuan Nguyen Gia, Behailu Negash, Arman Anzanpour, Iman Azimi, Mingzhe Jiang, and Pasi Liljeberg. "Exploiting smart e-health gateways at the edge of healthcare internet-of-things: a fog computing approach." Future Generation Computer Systems 78 (2018): 641-658. 
[8] Mukherjee, Mithun, Lei Shu, and Di Wang. "Survey of Fog Computing: Fundamental, Network Applications, and Research Challenges." IEEE Communications Surveys \& Tutorials (2018).

[9] Skourletopoulos, Georgios, Constandinos X. Mavromoustakis, George Mastorakis, Jordi Mongay Batalla, Ciprian Dobre, John N. Sahalos, Rossitza I. Goleva, and Nuno M. Garcia. "Game Theoretic Approaches in Mobile Cloud Computing Systems for Big Data Applications: A Systematic Literature Review." In Mobile Big Data, pp. 41-62. Springer, Cham, 2018.

[10] Lordan, Francesc, Daniele Lezzi, Jorge Ejarque, and Rosa M. Badia. "An Architecture for Programming Distributed Applications on Fog to Cloud Systems." In European Conference on Parallel Processing, pp. 325-337. Springer, Cham, 2017.

[11] Bose, Rajesh, Murari Krishna Saha, and Debabrata Sarddar. "Fog Computing Made Easy with the Help of Citrix and Billboard Manager." International Journal of Computer Applications 121, no. 7 (2015).

[12] Abbadi, Imad M. "Toward trustworthy clouds' internet scale critical infrastructure." In International Conference on Information Security Practice and Experience, pp. 71-82. Springer, Berlin, Heidelberg, 2011.

[13] Oppitz, Marcus, and Peter Tomsu. "Internet of Things." In Inventing the Cloud Century, pp. 435-469. Springer, Cham, 2018.

[14] Ray, Sandip, Yier Jin, and Arijit Raychowdhury. "The changing computing paradigm with internet of things: A tutorial introduction." IEEE Design \& Test 33, no. 2 (2016): 76-96.

[15] Laghari, Asif Ali, Hui He, Imtiaz A. Halepoto, M. Sulleman Memon, and Sajida Parveen. "Analysis of Quality of Experience Frameworks for Cloud Computing." IJCSNS 17, no. 12 (2017): 228.

[16] Seyyed Mohsen Hashemi,Amid Khatibi Bardsiri "Cloud Computing Vs. Grid Computing" ARPN Journal of Systems and Software, VOL. 2, NO.5, MAY 2012 ISSN 2222-9833.

[17] Laghari, Asif Ali, Hui He, Muhammad Shafiq, and Asiya Khan. "Application of Quality of Experience in Networked Services: Review, Trend \& Perspectives." Systemic Practice and Action Research (2018): $1-19$.

[18] Mell, Peter, and Tim Grance. "The NIST definition of cloud computing." (2011).

[19] Laghari, Asif Ali, Hui He, Muhammad Shafiq, and Asiya Khan. "Assessing effect of Cloud distance on end user's Quality of Experience (QoE)." In Computer and Communications (ICCC), 2016 2nd IEEE International Conference on, pp. 500-505. IEEE, 2016.

[20] Dempsey, David, and Felicity Kelliher. "Revenue Models and Pricing Strategies in the B2B SaaS Market." In Industry Trends in Cloud Computing, pp. 45-82. Palgrave Macmillan, Cham, 2018.

[21] Jagli, Dhanamma, Seema Purohit, and N. Subhash Chandra. "SAASQUAL: A Quality Model for Evaluating SaaS on the Cloud Computing Environment." In Big Data Analytics, pp. 429-437. Springer, Singapore, 2018.

[22] Krancher, Oliver, Pascal Luther, and Marc Jost. "Key Affordances of Platform-as-a-Service: SelfOrganization and Continuous Feedback." Journal of Management Information Systems 35 (2018): 1-43.

[23] Pahl, Claus. "Containerization and the paas cloud." IEEE Cloud Computing 2, no. 3 (2015): 24-31.

[24] Kashif, Ubaidullah Alias, Zulfiqar Ali Memon, Shafaq Siddiqui, Abdul Rasheed Balouch, and Rakhi Batra. "Architectural Design of Trusted Platform for IaaS Cloud Computing." International Journal of Cloud Applications and Computing (IJCAC) 8, no. 2 (2018): 47-65.

[25] Aggarwal, Ritu. "Resource Provisioning and Resource Allocation in Cloud Computing Environment." (2018).

[26] Briggs, Benjamin David, Lawrence A. Clevengen, Bartlet H. DeProspo, and Michael Rizzolo. "Structure, system, method, and recording medium of implementing a directed self-assembled security pattern." U.S. Patent Application 15/055,835, filed August 31, 2017.

[27] Saraswathi, M., and T. Bhuvaneswari. "A Secured Storage using AES Algorithm and Role Based Access in Cloud." (2017).

[28] Hu, Han, Yonggang Wen, and Dusit Niyato. "Public cloud storage-assisted mobile social video sharing: A supermodular game approach." IEEE journal on selected areas in communications 35, no. 3 (2017): 
545-556.

[29] Manu, A. R., V. K. Agrawal, and KN Bala Subramanya Murthy. "An empirical hunt for ally cooperative cloud computing utility." In Intelligent Systems and Control (ISCO), 2017 11th International Conference on, pp. 422-438. IEEE, 2017.

[30] Goyal, Sumit. "Public vs private vs hybrid vs community-cloud computing: A critical review." International Journal of Computer Network and Information Security 6, no. 3 (2014): 20.

[31] Ramanathan, Ramakrishnan, and B. Latha. "Towards optimal resource provisioning for HadoopMapReduce jobs using scale-out strategy and its performance analysis in private cloud environment." Cluster Computing (2018): 1-11.

[32] Li, Qing, Ze-yuan Wang, Wei-hua Li, Jun Li, Cheng Wang, and Rui-yang Du. "Applications integration in a hybrid cloud computing environment: Modelling and platform." Enterprise Information Systems 7, no. 3 (2013): 237-271.

[33] Huang, Dijiang, and Huijun Wu. Mobile Cloud Computing: Foundations and Service Models. Morgan Kaufmann, 2017.

[34] Mao, Yuyi, Changsheng You, Jun Zhang, Kaibin Huang, and Khaled B. Letaief. "A survey on mobile edge computing: The communication perspective." IEEE Communications Surveys \& Tutorials 19, no. 4 (2017): 2322-2358.

[35] Bonomi, Flavio, Rodolfo Milito, Preethi Natarajan, and Jiang Zhu. "Fog computing: A platform for internet of things and analytics." In Big data and internet of things: A roadmap for smart environments, pp. 169-186. Springer, Cham, 2014.

[36] Aazam, Mohammad, and Eui-Nam Huh. "Fog computing and smart gateway based communication for cloud of things." In Future Internet of Things and Cloud (FiCloud), 2014 International Conference on, pp. 464-470. IEEE, 2014.

[37] Aazam, Mohammad, and Eui-Nam Huh. "Fog computing micro datacenter based dynamic resource estimation and pricing model for IoT." In Advanced Information Networking and Applications (AINA), 2015 IEEE 29th International Conference on, pp. 687-694. IEEE, 2015.

[38] Gupta, Harshit, Amir Vahid Dastjerdi, Soumya K. Ghosh, and Rajkumar Buyya. "iFogSim: A toolkit for modeling and simulation of resource management techniques in the Internet of Things, Edge and Fog computing environments." Software: Practice and Experience 47, no. 9 (2017): 1275-1296.

[39] Saurez, Enrique, Harshit Gupta, Ruben Mayer, and Umakishore Ramachandran. "Demo abstract: Fog computing for improving user application interaction and context awareness." In Internet-of-Things Design and Implementation (IoTDI), 2017 IEEE/ACM Second International Conference on, pp. 281-282. IEEE, 2017.

[40] Osanaiye, Opeyemi, Shuo Chen, Zheng Yan, Rongxing Lu, Kim-Kwang Raymond Choo, and Mqhele Dlodlo. "From cloud to fog computing: A review and a conceptual live VM migration framework." IEEE Access 5 (2017): 8284-8300.

[41] https://www.cisco.com/c/en/us/solutions/internet-of-things/iot-fog-applications.html

[42] Wai, Ei Nyein Chan, Pei-Wei Tsai, and Jeng-Shyang Pan. "Hierarchical PSO clustering on mapreduce for scalable privacy preservation in big data." In International Conference on Genetic and Evolutionary Computing, pp. 36-44. Springer, Cham, 2016.

[43] ZHENGKUI, WANG. "Scalable Data Analysis on MapReduce-based Systems." PhD diss., 2013.

[44] Shrivastava, Sonika, and R. K. Pateriya. "Secure Framework for E-Commerce Applications in Cloud Environment." In Improving E-Commerce Web Applications Through Business Intelligence Techniques, pp. 82-109. IGI Global, 2018.

[45] Raj, R. Sundar, and Dr V. Murali Bhaskaran. "Comparative Analysis of Cloud Tools to Implement Automated Resource and Security Management." Indian Journal of Engineering (An International Journal) 14, no. 35 (2017): 20-32.

[46] Gupta, Harshit, Amir Vahid Dastjerdi, Soumya K. Ghosh, and Rajkumar Buyya. "iFogSim: A toolkit for modeling and simulation of resource management techniques in the Internet of Things, Edge and Fog 
computing environments." Software: Practice and Experience 47, no. 9 (2017): 1275-1296.

[47] http://www.cloudbus.org/fog/

[48] https://github.com/Cloudslab/iFogSim

[49] https://fogproject.org/

[50] Calheiros, Rodrigo N., Rajiv Ranjan, Anton Beloglazov, César AF De Rose, and Rajkumar Buyya. "CloudSim: a toolkit for modeling and simulation of cloud computing environments and evaluation of resource provisioning algorithms." Software: Practice and experience 41, no. 1 (2011): 23-50.

[51] https://www.zenoss.com/

[52] Hendrick, Evan, Brad Schooley, and Chunming Gao. "CloudHealth: developing a reliable cloud platform for healthcare applications." In Consumer Communications and Networking Conference (CCNC), 2013 IEEE, pp. 887-891. IEEE, 2013.

[53] "Cloudera", http://www.cloudera.com.

[54] Laghari, Asif Ali, Hui He, Muhammad Shafiq, and Asiya Khan. "Impact of storage of mobile on quality of experience (QoE) at user level accessing cloud." In Communication Software and Networks (ICCSN), 2017 IEEE 9th International Conference on, pp. 1402-1409. IEEE, 2017.

[55] Laghari, Asif Ali, Hui He, Shahid Karim, Himat Ali Shah, and Nabin Kumar Karn. "Quality of Experience Assessment of Video Quality in Social Clouds." Wireless Communications and Mobile Computing 2017 (2017).

[56] Yi, Shanhe, Cheng Li, and Qun Li. "A survey of fog computing: concepts, applications and issues." In Proceedings of the 2015 workshop on mobile big data, pp. 37-42. ACM, 2015.

[57] Yi, Shanhe, Zhengrui Qin, and Qun Li. "Security and privacy issues of fog computing: A survey." In International conference on wireless algorithms, systems, and applications, pp. 685-695. Springer, Cham, 2015.

[58] Laghari, Asif Ali, Hui He, and Muhammad Ibrahim Channa. "Measuring effect of packet reordering on quality of experience (QoE) in video streaming." 3D Research 9, no. 3 (2018): 30.

[59] Laghari, Asif Ali, Hui He, Asiya Khan, and Sajida Karim. "Impact of Video File Format on Quality of Experience (QoE) of Multimedia Content." 3D Research 9, no. 3 (2018): 39.

[60] Yadav, Rahul, Weizhe Zhang, Huang Chen, and Tao Guo. "MuMs: Energy-Aware VM Selection Scheme for Cloud Data Center." In Database and Expert Systems Applications (DEXA), 2017 28th International Workshop on, pp. 132-136. IEEE, 2017.

[61] Yadav, Rahul, and Weizhe Zhang. "MeReg: Managing Energy-SLA Tradeoff for Green Mobile Cloud Computing." Wireless Communications and Mobile Computing 2017 (2017).

[62] Laghari, A., H. He, A. I. Khan, N. Kumar, and R. Kharel. "Quality of Experience Framework for Cloud Computing (QoC)." (2018). August 2018, IEEE Access, DOI: 10.1109/ACCESS.2018.2865967

[63] Laghari, Asif Ali, Hui He, Muhammad Shafiq, and Asiya Khan. "Assessment of quality of experience (QoE) of image compression in social cloud computing." Multiagent and Grid Systems 14, no. 2 (2018): 125-143.

\section{Authors' Profiles}

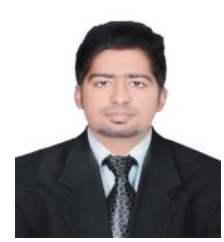

Vishal Kumar received the B.E degree from the Quaid-e -Awam University of Engineering Science And Technology Nawabshah, Pakistan, in February 2016, he joined the school of Astronautics in department of control science and engineering in September 2016, at Harbin Institute of Technology, China where he is now a Masters student. He has published International conference paper in ICCAR EI conference proceedings. His Current research interests include Computer networks, cloud computing, and computer vision system. 


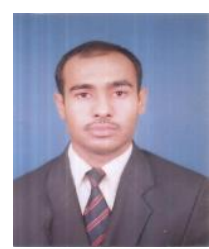

Asif Ali Laghari received the B.S. degree in Information Technology from the Quaid-e-Awam University of Engineering Science and Technology Nawabshah, Pakistan, in 2007 and Master degree in Information Technology from the QUEST Nawabshah Pakistan in 2014. From 2007 to 2008, he was a Lecturer in the Computer and Information Science Department, Digital Institute of Information Technology, Pakistan. In 2015, he joined the school of the Computer Science \& Technology, Harbin Institute of Technology, where he is now a PhD student. He has published more than 10 technical articles in scientific journals and conference proceedings. His current research interests include Computer networks, cloud computing, and multimedia QoE management.

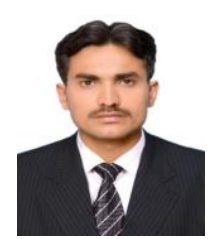

Shahid Karim received his BS degree in electronics from Comsats Institute of Information Technology, Abbottabad, Pakistan, and his MS degree in electronics and information engineering from Xi'an Jiaotong University, China, in 2010 and 2015, respectively. He is currently pursuing his $\mathrm{PhD}$ at the Department of Information and Communication Engineering, School of Electronics and Information Engineering, Harbin Institute of Technology (HIT), China. His current research interests include image processing, object detection, and classification toward optical remote sensing imagery.

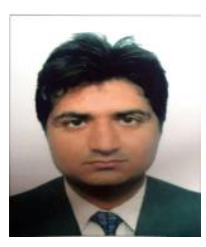

Muhammad Shakir received his B.E degree in computer systems engineering from Quaid-e Awam University of engineering science and technology Nawabshah, Pakistan in Feb 2016. In September 2016 he joined the school of Astronautics major in control science and engineering at Harbin Institute of technology where he is now a master's student. He has published two technical articles, conference proceedings. His current research interest include cloud computing and data driven fault diagnosis.

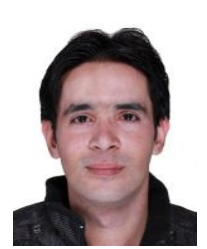

Ali Anwar Brohi received B.S. degree in Mechanical Engineering from Mehran University of Engineering and Technology, SZAB Campus, Khairpur Mir's, Sindh, Pakistan in 2010 and MS degree in Mechanical Engineering from Harbin Institute of Technology, Harbin, China in 2015. $\mathrm{He}$ is currently working toward a Ph.D. degree in the School of Energy Science and Engineering at Harbin Institute of Technology, Harbin, China. His research interests include entropy generation, heat transfer and fluid dynamics.

How to cite this paper: Vishal Kumar, Asif Ali Laghari, Shahid Karim, Muhammad Shakir, Ali Anwar Brohi,"Comparison of Fog Computing \& Cloud Computing", International Journal of Mathematical Sciences and Computing (IJMSC), Vol.5, No.1, pp.31-41, 2019.DOI: 10.5815/ijmsc.2019.01.03 